\title{
New Analytical Formulae to Calibrate Well-type Scintillation Detectors Efficiency
}

\author{
Abbas $\mathrm{MI}^{1 *}$, Ibrahim $\mathrm{OA}^{2}$, Ibrahim $\mathrm{T}^{1,2}$ and Sakr $\mathbf{M}^{1,2}$ \\ ${ }^{1}$ Department of Physics, Faculty of Science, Alexandria University, Egypt \\ ${ }^{2}$ Department of Physics, Faculty of Science, Beirut Arab University, Lebanon
}

\begin{abstract}
Direct Mathematical calculations for the absolute efficiency of well-type gamma ray scintillation crystals are described. Calculated detection efficiencies are drawn for two commonly used well crystals. Comparisons are made between present works and published Monte Carlo values. The present approach proved quite success in predicting the efficiency of well type detectors providing only the geometry and materials of the system formed of source, detector and shielding as well as the energy of the emitted photons.
\end{abstract}

Keywords: New mathematical method; Gamma-spectroscopy; Absolute efficiency; Well-type detector

\section{Introduction}

Direct mathematical method to calculate the efficiency of radioactive systems was first proposed by Selim and Abbas and then it was used by several authors [1-12]. The comparison between the calculated values of efficiencies and experimental ones provided in these papers shows that the error is very small. This method proves its accuracy and success for many detector geometries; like: coaxial detectors [14], parallelepiped detectors [5] and borehole detectors [12]; and types like: semiconductors [4-7] and scintillators [1-3] Besides, the direct mathematical method is built on simple mathematical approach and it does not need long computer programs nor previous experimental calculation of the efficiency of standard source like other Monte Carlo method [13-16]. In this work we used direct mathematical method to predict the efficiency of two well type detectors, in detecting the gamma photons produced by sources placed in their well cavities, over wide range of photon energy for seven different source geometries and positions including point sources with very small dimensions and cylindrical sources. These detectors are scintillators and their active material is Sodium Iodide (NaI). Sodium Iodide is widely used in detecting of gamma photons nowadays because of the high efficiency of these crystals, relatively low cost and availability in different shapes and sizes. In our calculation, the effect of beam attenuation caused by well's walls and bottom is included as well as self-attenuation caused by the material of cylindrical sources.

For each source geometry, a comparison between our calculated value of efficiencies using this approach and reference values obtained from Pomme et al. [15] is given. This comparison shows that our values were in good agreement with Pomme et al. [15], proving the success of our approach in predicting the efficiencies of these detectors over wide range of photon energy.

The arrangement of this paper is as follows: providing a general explanation of the direct mathematical method in section 2, geometric description of well type detector is given in section 3. Detailed explanation of the mathematical perspective used is done. Comparison between calculated and reference values of efficiency is done in seven independent figures. Finally the conclusion is presented.

\section{Direct Mathematical Method}

This method is theoretical based on the following fundamentals:
When a photon with certain energy strikes a detector without absorbing walls or windows, the probability for it to interact with this detector and depose energy to be recorded is:

$$
f=1-e^{-\mu . d}
$$

Where, $\mu$ represents the total attenuation coefficient of the detector active volume, and $d$, is the photon path length traveled through the detector active volume. The factor determining the beam attenuation due the source container and the detector end cap materials is called the attenuation factor, $F_{a t t}$. So the probability is rewritten as:

$$
f_{j}=F_{a t t}\left(1-e^{-\mu . d}\right)
$$

The attenuation factor $F_{a t t}$, is expressed as:

$$
F_{\text {att }}=e^{-\sum_{i} \mu_{i} \delta_{i}}
$$

Where, $\mu_{i}$ is the attenuation coefficient of the $i^{\text {th }}$ absorber for a gamma photon with energy $E_{\gamma}$ and $\delta_{i}$ is the gamma photon path length through the $i^{\text {th }}$ absorber.

\section{Geometric Description of Well Type Detector}

The detector, we calculated its efficiency, is a well type $\mathrm{NaI}$ detector of cylindrical shape. This detector is illustrated in Figure 1. The total height of this detector is marked as " $\mathrm{T}$ " in the mentioned figure and its total radius "R". This detector contains a coaxial cavity (well) of cylindrical shape also that has a radius $r$ and a height $\mathrm{D}$ as shown in Figure 1. A small radioactive source can be placed in the well coaxial cavity. The source might be a small cylinder that can fit in the cavity or an extremely small source (point source). This source contains usually radioactive nuclei that undergo beta or alpha decay, and then emit gamma photons.

*Corresponding author: Abbas MI, Department of Physics, Faculty of Science, Alexandria University, Egypt, Tel: 203 5921675; E-mail: mabbas@physicist.net

Received February 03, 2016; Accepted April 14, 2016; Published April 20, 2016

Citation: Abbas MI, Ibrahim OA, Ibrahim T, Sakr M (2016) New Analytical Formulae to Calibrate Well-type Scintillation Detectors Efficiency. J Phys Math 7: 164. doi:10.4172/2090-0902.1000164

Copyright: ( 2016 Abbas MI, et al. This is an open-access article distributed under the terms of the Creative Commons Attribution License, which permits unrestricted use, distribution, and reproduction in any medium, provided the original author and source are credited. 


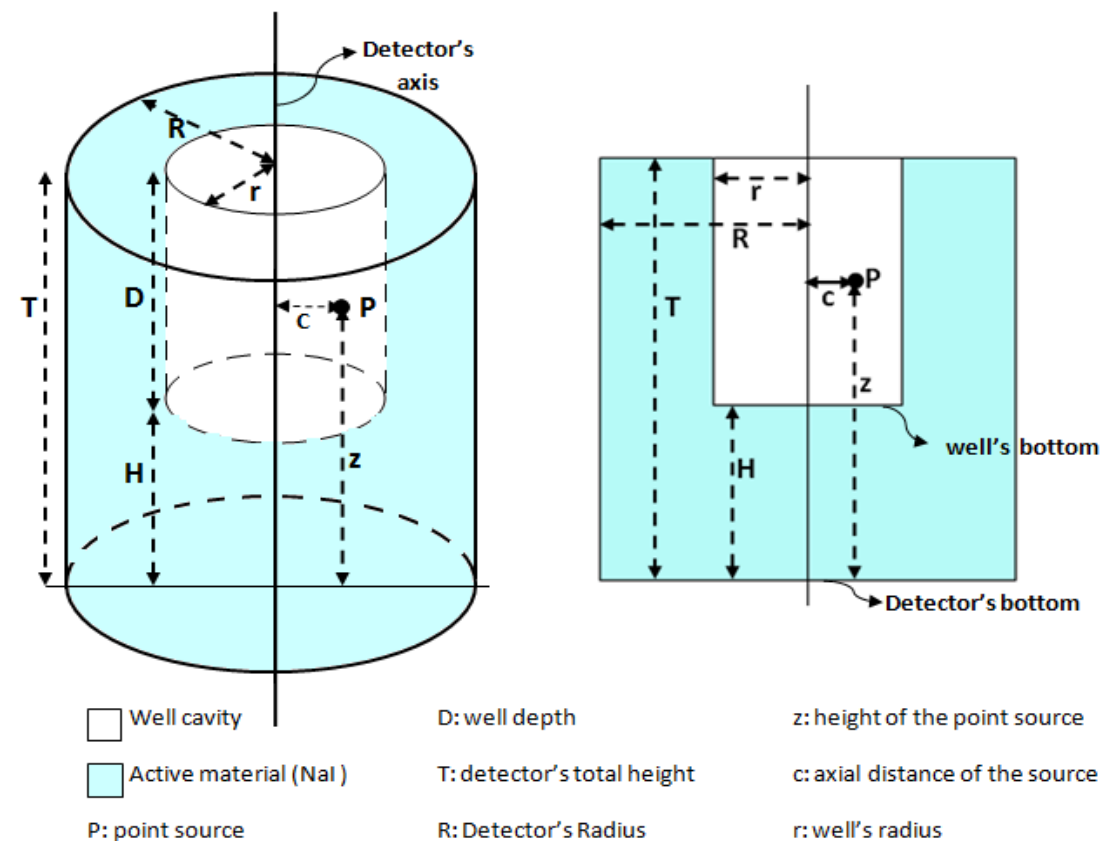

Figure 1: Three dimensional sketches and a vertical section view for a well type detector.

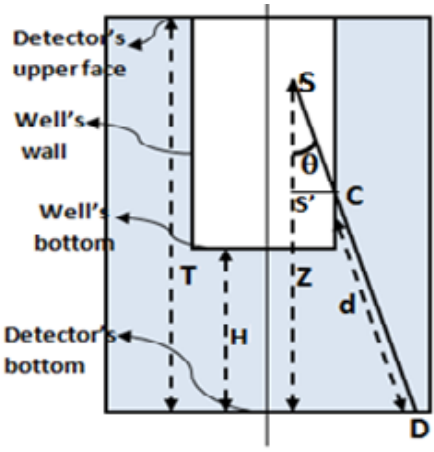

$\square$ Well Cavity

$\theta$ : polar angle

$\varphi$ : azimuth angle

r: well's radius

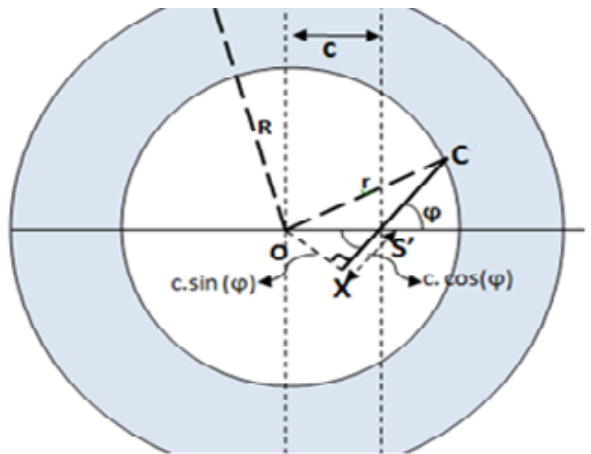

Active material(Nal)

S: point source

c: distance from axis to $S$

d: distance covered in $\mathrm{Nal}$

" $\mathrm{C}$ ": the point where the photon enters the active material.

" $\mathrm{O}$ ": Axial point belonging to the horizontal plane containing " $\mathrm{C}$ "

"D": the point where the photon leaves the detector.

" $X$ ": the foot of perpendicular from $O$ to $S^{\prime} C$

Figure 2: Three dimensional schematic diagram of a well type detector (left) and to a horizontal view plane (right) to illustrate the path length function $\mathrm{d}_{2}$.

\section{Mathematical Perspective}

\section{Path-lengths}

There are five different path lengths "faiths" $\left(d_{1}, d_{2}, d_{3}, d_{4}\right.$ and $\left.d_{5}\right)$ can be followed by a photon in our system depending on where the photon enters the active material and leaves it, the photon can enter either from well's bottom or the well's side and leaves either from detector bottom, upper face or outer side.

If the photon enters the active material from the bottom and leaves from the bottom: Just by making projection on the axis of the detector, one can derive the function of path length to be:

$$
d_{1}=\frac{H}{\cos (\theta)}
$$

If the photon enters the active material from well's side and leaves from detector's bottom: By making projections on a vertical and horizontal planes (Figure 2). We can derive the path length function of the distance covered in the active material (NaI) in terms of the detector and source dimensions shown in Figure 1, as well as the polar and azimuth angle. As shown in Figure 2. If the photon enters from the side of the well cavity and leaves from the detector's bottom, the distance it covered inside the detecting material $(\mathrm{NaI})$ is labeled by $\mathrm{CD}$ (d) in the figure, the distance $\overline{C D}=\overline{S D}-\overline{S C}$ where $\overline{S D}$ can 
be taken from the vertical view (left) to be $\mathrm{z} / \cos (\theta)$, and SC is simply $S^{\prime} \mathrm{C} / \sin (\theta)$. The horizontal view to the right of the same figure. Figure 2 shows the triangle $\mathrm{OXC}$ right at $\mathrm{X}$, the simple geometry of $\mathrm{OXC}$ gives $\overline{C X}=\sqrt{O C^{2}-O X^{2}}$ and $\overline{\mathrm{CS}^{\prime}}=\overline{C X}-\overline{\mathrm{XS}^{\prime}}$; but $\overline{\mathrm{OX}}=c \cdot \sin (\varphi)$ and $\overline{\mathrm{XS}^{\prime}}=c \cdot \cos (\varphi)$ and $\overline{O C}=\mathrm{r}$. So $\mathrm{CS}^{\prime}=\sqrt{r^{2}-(c \cdot \sin (\varphi))^{2}}$ the path length covered by a photon entering from the well's side and leaving from the detector's bottom will be:

$$
d_{2}=\frac{z}{\cos (\theta)}-\frac{ \pm \mathrm{c} \cdot \cos (\varphi)+\sqrt{\mathrm{r}^{2}-(\mathrm{c} \cdot \sin \varphi)^{2}}}{\sin (\theta)}
$$

To discuss the " \pm " sign in the equation of path length $\mathrm{d}_{2}$, we consider the projection on a horizontal plane containing the point where the photon enters the active material shown in Figure 3, the photon can enter the active material from the nearer arc shown as dashed arc in the figure or from the farther arc shown as solid arc. If it enters from the nearer arc (left part of Figure 3), the distance will be $\overline{\mathrm{S}^{\prime} \mathrm{A}}=\overline{\mathrm{XA}}-\overline{\mathrm{XS}^{\prime}}$ and thus $\overline{S^{\prime} A}=\sqrt{r^{2}-(c \cdot \sin (\varphi))^{2}}$ If the photon enters from the farther $\operatorname{arc}$ (right part of the figure), the distance $\overline{\mathrm{S}^{\prime} \mathrm{B}}$ in the figure is done by $\overline{\mathrm{S}^{\prime} \mathrm{B}}=\overline{X B}+\overline{S^{\prime} X}=\sqrt{\mathrm{r}^{2}-(\mathrm{c} \cdot \sin (\varphi))^{2}}+c \cdot \cos (\phi)$

If the photon enters the active material from well's bottom and leaves from the outer side: As shown in Figure 4, if the photon enters from the well's bottom and leaves from detector's outer side the distance covered inside the active material is $\overline{\mathrm{AB}}$ where $\overline{\mathrm{AB}}=\overline{\mathrm{SB}}-\overline{\mathrm{SA}} \mathrm{SA}$ is clearly $\mathrm{f} / \cos (\theta)$ and $\overline{\mathrm{SB}}=\overline{\mathrm{S}^{\prime} \mathrm{B}} / \sin (\theta)$. To derive $\mathrm{S}^{\prime} \mathrm{B}$ with respect to $\mathrm{R}$, and $\varphi$, the horizontal view to the right is done, $\overline{\mathrm{S}^{\prime} \mathrm{B}}=\overline{B X}+\overline{X S^{\prime}}$ here $\overline{B X}=\sqrt{r^{2}-(c \cdot \sin (\varphi))^{2}}$ and $\overline{X S^{\prime}}=c \cdot \cos (\varphi)$

So $\overline{S B}=\frac{-\mathrm{c} \cdot \cos (\varphi)+\sqrt{\mathrm{R}^{2}-(\mathrm{c} \cdot \sin \varphi)^{2}}}{\sin \theta}$ and the path length
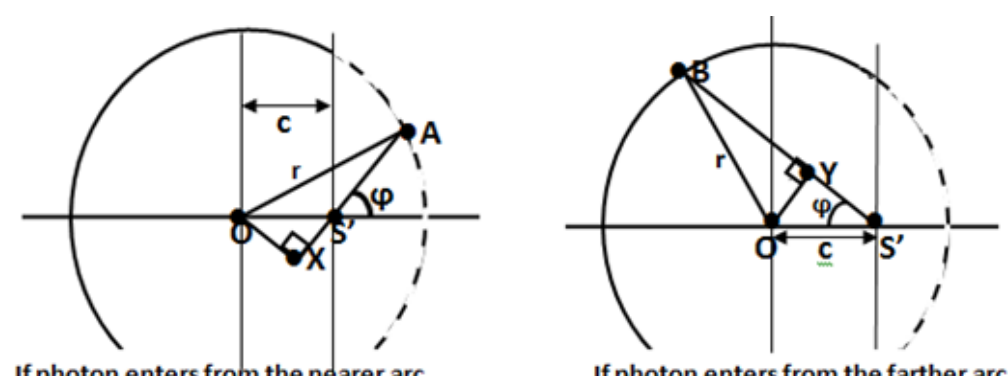

If photon enters from the farther arc

\begin{tabular}{|c|c|c|}
\hline OA: Radius of well ( $r$ ) & $\varphi$ : azimuth Angle & $-{ }^{\text {Nearerarc }}$ \\
\hline OB: Radius of well (r) & $S^{\prime}:$ projection of S (source) & Fartherarc \\
\hline$\overline{O X}: \operatorname{c.sin}(\varphi)$ & $\overline{S^{\prime} X}: \cos \cdot \cos (\varphi)$ & $\overline{S^{\prime} Y}: c \cdot \cos (\varphi)$ \\
\hline$\overline{O Y}: c \cdot \sin (\varphi)$ & $\overline{X A}=\sqrt{r^{2}-(c \cdot \sin (\varphi))^{2}}$ & $\overline{Y B}=\sqrt{r^{2}-(c \cdot \sin (\varphi))^{2}}$ \\
\hline$\overline{S^{\prime} A}=\sqrt{r^{2}-(c \cdot \sin (\varphi)}$ & $-c \cdot \cos (\varphi)$ & $\overline{(c \cdot \sin (\varphi))^{2}}+c \cdot \cos (\varphi)$ \\
\hline \multicolumn{3}{|c|}{ “c": distance between detector' axis and point source } \\
\hline \multicolumn{3}{|c|}{ "A": point where the photon enters the material from the nearer arc, } \\
\hline \multicolumn{3}{|c|}{ " $\mathrm{B}$ ": point where the photon enters the material from the farther arc. } \\
\hline \multicolumn{3}{|c|}{ "O": Axial point belongs to the horizontal plane containing A or B. } \\
\hline \multicolumn{3}{|c|}{ "X": foot of perpendicular from $O$ to $S^{\prime} A$ or $S^{\prime} B$} \\
\hline
\end{tabular}

Figure 3: Horizontal View made to discuss the " \pm " sign in the equation of the path length: $d_{2}$. function " $\mathrm{d}_{3}$ " in this case will be:

$$
d_{3}=\frac{ \pm \mathrm{c} \cdot \cos (\varphi)+\sqrt{\mathrm{R}^{2}-(\mathrm{c} \cdot \sin \varphi)^{2}}}{\sin \theta}-\frac{\mathrm{f}}{\cos \theta}
$$

If the photon enters the active material from the well's wall and leaves from the outer wall: If the photon enters from the well's wall, the total distance it covers inside the well cavity is $\frac{ \pm c \cdot \cos (\varphi)+\sqrt{r^{2}-(c \cdot \sin \varphi)^{2}}}{\sin \theta}$ (derived previously to be used in equation 5). While if it leaves from the detector's outer side, the distance it covers in the entire system (active material + well) is $\frac{ \pm c \cdot \cos (\varphi)+\sqrt{r^{2}-(c \cdot \sin \varphi)^{2}}}{\sin \theta}$ (derived previously to be used in equation 6). So the distance covered in the active material will be:

$$
d_{4}=\frac{ \pm \mathrm{c} \cdot \cos (\varphi)+\sqrt{\mathrm{R}^{2}-(\mathrm{c} \cdot \sin \varphi)^{2}}}{\sin \theta}-\frac{ \pm \mathrm{c} \cdot \cos (\varphi)+\sqrt{\mathrm{r}^{2}-(\mathrm{c} \cdot \sin \varphi)^{2}}}{\sin \theta}
$$

If the photon enters the active material from the inner side and leaves from the upper surface: To derive the path length function $\mathrm{d}_{5}$; Figure 5 shows three dimensional diagram for a photon entering from well's wall and leaving from detector's upper surface the total distance the photon covers in the entire system(well and active material) labeled as $\mathrm{SF}$ in the figure is $\frac{T-z}{\cos (\pi-\theta)}$ while the distance it covers in the well is $\frac{\overline{S^{\prime} E}}{\sin (\pi-\theta)} S^{\prime} E$ can be derived using the horizontal view in the same figure $\overline{S^{\prime} E}=\overline{S^{\prime} H^{\prime}}+\overline{H^{\prime} E}$ So $\overline{S^{\prime} E}=$ c. $\cos (\varphi)+\sqrt{\mathrm{r}^{2}-(\mathrm{c} \cdot \sin \varphi)^{2}}$

$$
d_{5}=\frac{T-z}{\cos (\pi-\theta)}-\frac{ \pm \mathrm{c} \cdot \cos (\varphi)+\sqrt{\mathrm{r}^{2}-(\mathrm{c} \cdot \sin \varphi)^{2}}}{\sin (\pi-\theta)}
$$




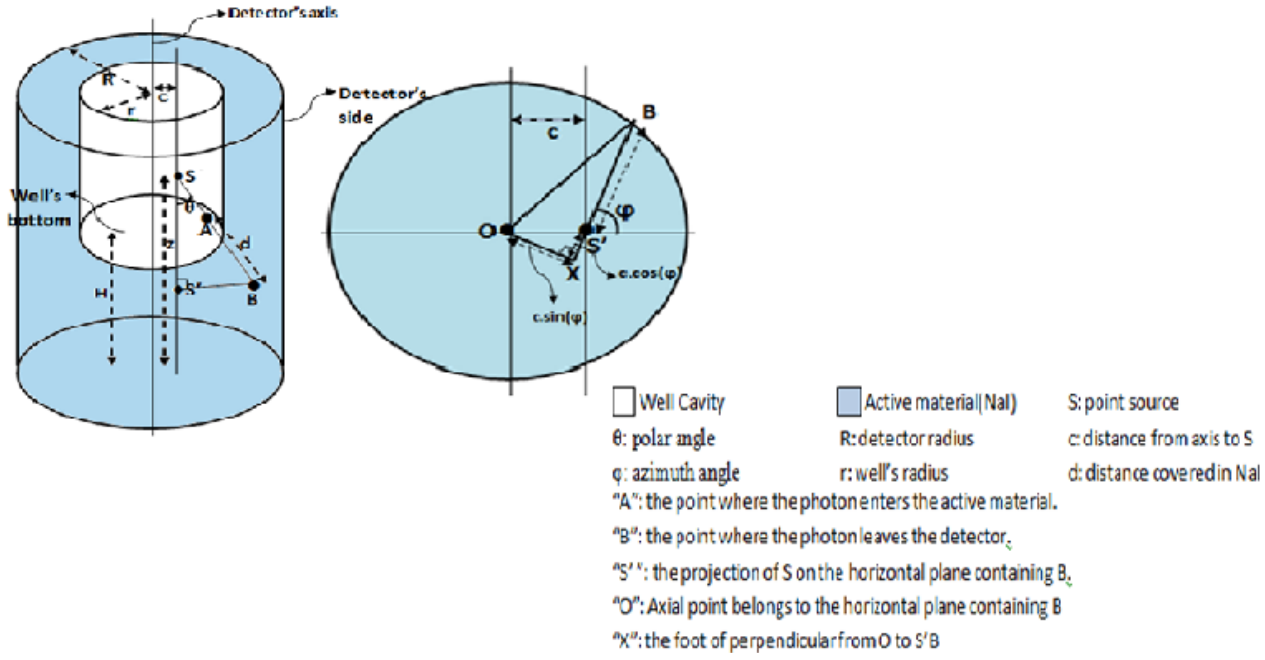

Figure 4: Three dimensional sketch and horizontal view made to derive the path length function: $d_{3}$.

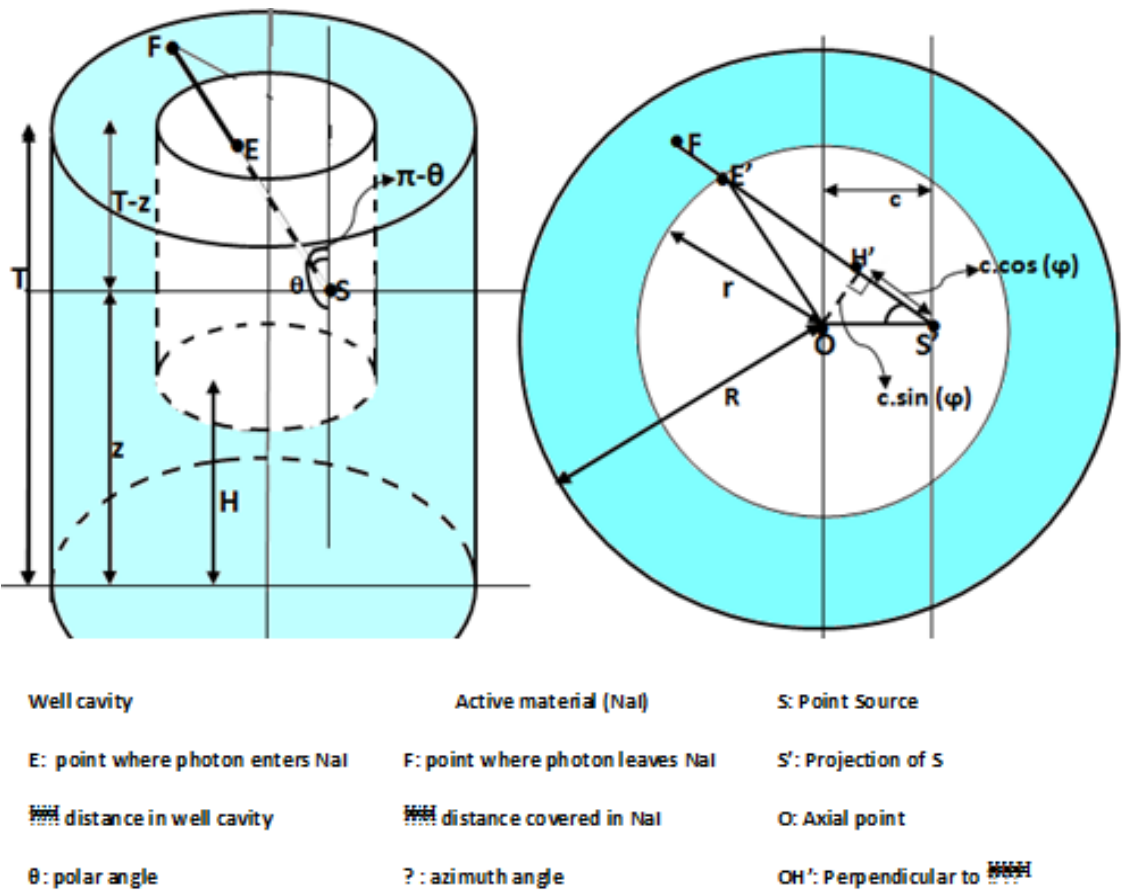

Figure 5: Three dimensional diagrams (left) and a projection to the plane containing the point $\mathrm{E}$ where the photon enters the active material (Nal).

Note: for an axial point source all items multiplied by " $\mathrm{c}$ " in the previous equations tend to zero.

\section{Total efficiency of an axial point source}

By definition, the efficiency of a source - detector system is the number of photons detected divided by the number of photons emitted. So, taking the total solid angle to be $4 \pi$, and using equation (2) we obtain:

$$
\varepsilon=\frac{1}{4 \pi} \int_{0}^{\pi 2 \pi} \int_{0}^{2 \pi} \sin (\theta) d \varphi d \theta
$$

Where, $f_{j}=F_{a t t}\left(1-e^{-\mu . d j}\right)(j \rightarrow 1-5) \mathrm{d}_{j}$ can be one of the five functions described before in section 4.1. So to develop equation 9 we just want to replace $d_{j}(j \rightarrow 1-5)$ by its function and we integrate over the entire solid angle taking into account that it might depend on both $\varphi$ and $\theta$. But in the case of an axial point source, there is symmetry on the azimuth angle $\varphi$. We have to distinguish between two cases

If the axial point source is located at a height, $z>\frac{H}{R-r} . R$ (Figure 6, right):

$$
\begin{aligned}
& \varepsilon=\frac{1}{4 \pi}\left(\int_{0}^{\theta_{1} 2 \pi} \int_{0} f_{1} d \varphi d \theta+\int_{\theta_{1}}^{\theta_{2} 2 \pi} \int_{0} f_{3} d \varphi d \theta+\int_{\theta_{2}}^{\theta_{2} 2 \pi} f_{0} d \varphi d \theta+\int_{\theta_{3}}^{\theta_{4} 2 \pi} \int_{0} f_{5} d \varphi d \theta\right) \\
& \theta_{1}=\tan ^{-1}\left(\frac{R}{z}\right) ; \quad \theta_{2}=\tan ^{-1}\left(\frac{r}{z-h}\right) ; \quad \theta_{3}=\pi-\tan ^{-1}\left(\frac{R}{H-z}\right) ;
\end{aligned}
$$




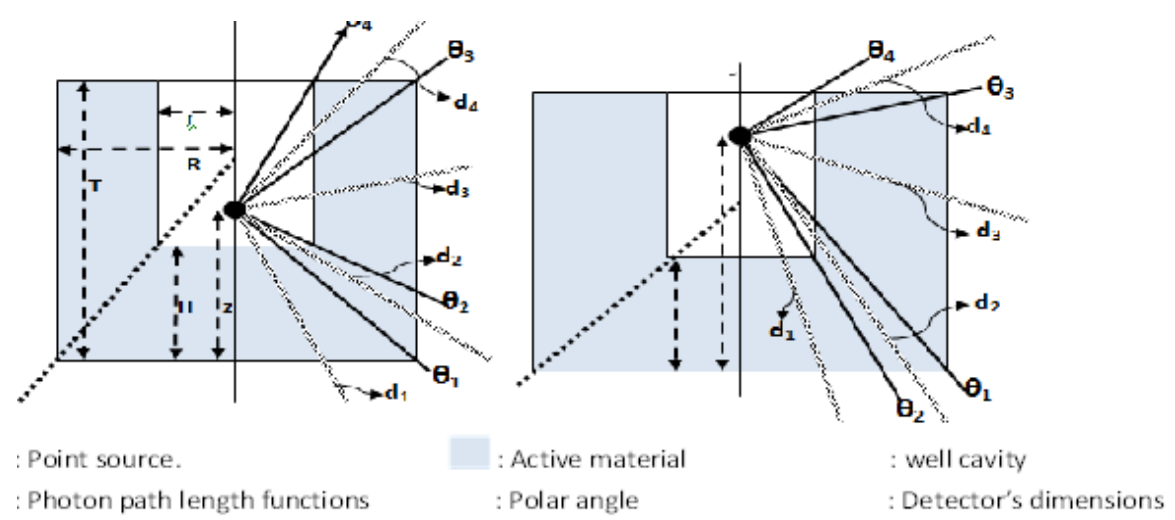

Figure 6: Vertical section view of the detector with an axial point source in two cases: Case 1 shows a lower point source below with $z<H /(R-r) . R($ left). Case 2: higher point source with $z>H /(R-r) \cdot R$ (to the right). Note: $z, R, H$ and $r$ were defined in Figure 1.

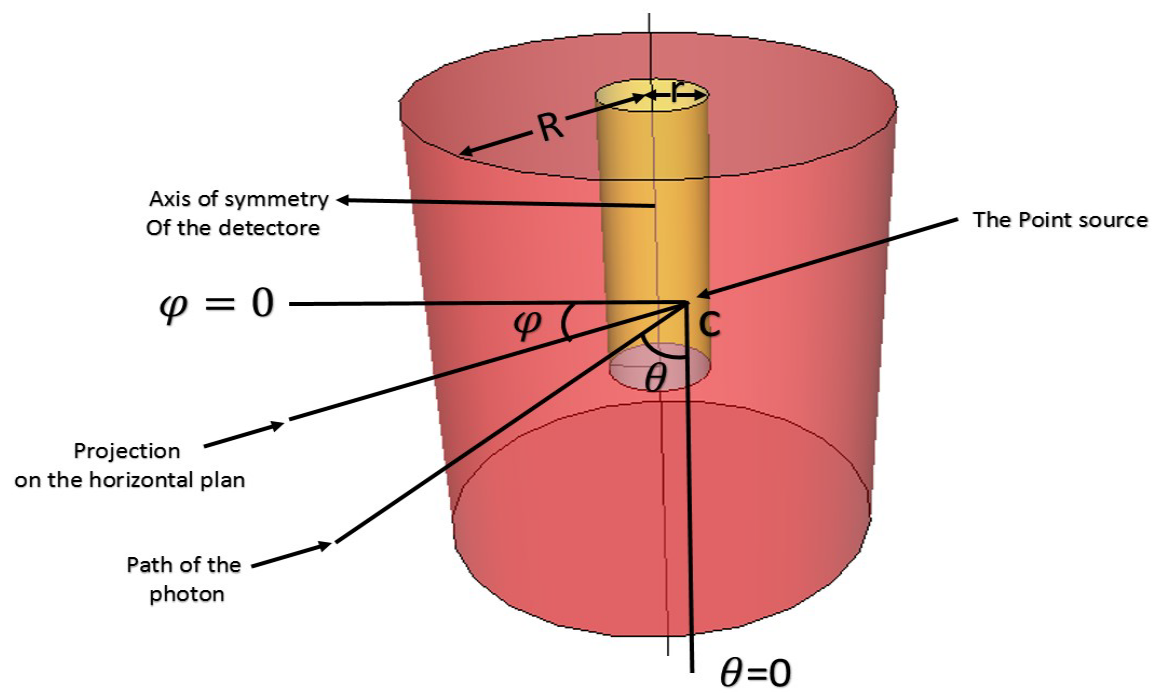

Figure 7a: Shows a 3D- drawing showing the references of the polar and azimuthal angles.

$\theta_{4}=\pi-\tan ^{-1}\left(\frac{r}{H-z}\right)$

If the axial point source is located at a height, $z<\frac{\mathbf{H}}{\mathbf{R}-\mathbf{r}} . \mathbf{R}$ (refer to Figure 6, left): applying the equation (2) gives:

$$
\varepsilon=\frac{1}{4 \pi}\left(\int_{0}^{\theta_{2}} \int_{0}^{2 \pi} f_{1} d \varphi d \theta+\int_{\theta_{2}}^{\theta_{1}} \int_{0}^{2 \pi} f_{2} d \varphi d \theta+\int_{\theta_{1}}^{\theta_{3} 2 \pi} f_{0}^{2 \pi} f_{4} d \varphi d \theta+\int_{\theta_{3}}^{\theta_{4} 2 \pi} \int_{0}^{2 \pi} f_{5} d \varphi d \theta\right)
$$

\section{Total efficiency of a non-axial point source}

For a non-axial point source one can calculate the total efficiency by applying equation 9 , but first we have to take references for the azimuth angle " $\varphi$ " as well as for the polar angle " $\theta$ ". Since each point should belong to certain diameter we take the diameter containing the point-source as the reference of azimuthal angle " $\varphi=0$ " in order to obtain certain symmetry in the Figure $7 \mathrm{a}$. As well as we take the line parallel to the detector axis passing by the point source is the reference of polar angle " $\theta=0$ ". The plane passing by the point source and containing the axis of the detector as well as the horizontal line having $\varphi=0$; can be considered as plane of reference in our case because it cuts the detector into two identical parts. We divide the detector into four zones as indicated in Figure $7 \mathrm{~b}$. The aim of this technique is to make the equations easier. The two regions to the right are considered closer to the source, so the signs $( \pm)$ in the equations $(6-8)$ will turn to $(-)$. Figure 7 a shows a $3 \mathrm{D}$ - drawing showing the references of the polar and azimuthal angles.

Zone $A$, the point is closer to the detector axis and $0<\theta<\pi / 2$ : To derive the mathematical equations, we will know first the photon path lengths allowed in this zone. Here the photon can enter either from the well cavity bottom or side and leave either from the outer side or bottom of detector. Thus, four path lengths will be allowed in this case which are: $d_{1}, d_{2}, d_{3}$ and $d_{4}$ (Tables 1 and 2 ). In this section we will study the different faiths when the photon enters the detector, as well as the different faiths when this photon leaves the detector, then we combine them to find different faiths of photon path length in the detector active volume. We will do the same strategy for the other zones. First we will study how the photon leaves the detector, we will make a projection on a horizontal plane containing the bottom of the detector so, as illustrated in Figure 8, we obtain:

- For $\theta<\theta_{1}$; the photon must leave from the bottom.

- For $\theta_{1}<\theta<\theta_{2}$; the photon can leave from the side up to certain azimuth angle $\varphi_{\mathrm{a}}$, while it leaves from the bottom when $\varphi>\varphi_{\mathrm{a}}$. 


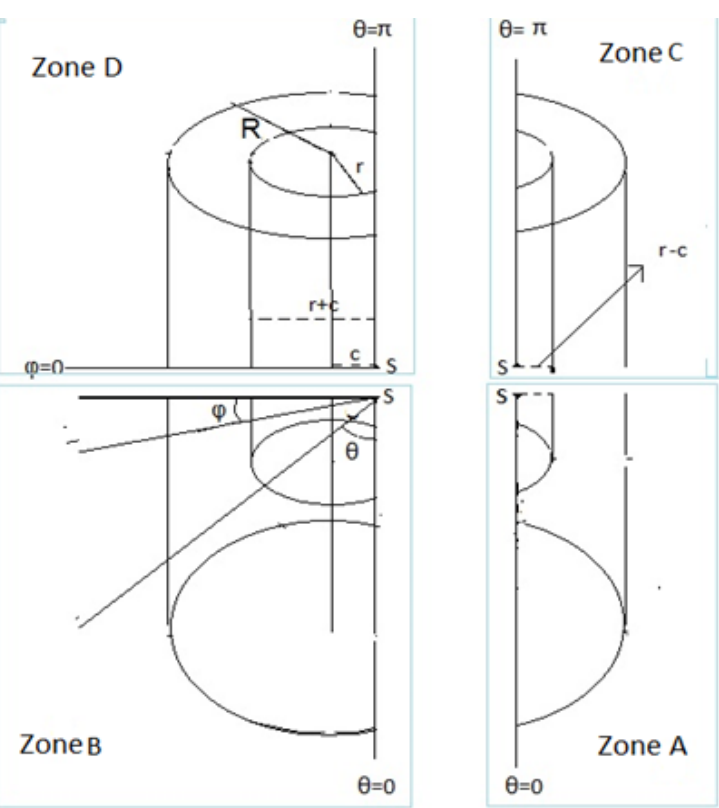

Figure 7b: Shows the decomposition of our system into four different zones.

\begin{tabular}{|c|c|c|c|c|}
\hline $\begin{array}{l}\text { The proton leaves } \\
\text { from }\end{array}$ & $\theta<\theta_{1}$ bottom & $\begin{array}{r}\theta_{1}<\theta<\theta_{2} \\
\varphi<\varphi_{\mathrm{a}} \text { side }\end{array}$ & $\begin{array}{c}\theta_{1}<\theta<\theta_{2} \\
\varphi>\varphi_{\mathrm{a}} \text { bottom }\end{array}$ & $\theta>\theta_{2}$ side \\
\hline$\theta<\theta_{3}$ the well cavity bottom & $\mathrm{d}_{1}$ & $d_{3}$ & $\mathrm{~d}_{1}$ & $d_{3}$ \\
\hline $\begin{array}{l}\theta_{3}<\theta<\theta_{4} \text { and } \varphi<\varphi_{a}^{\prime} \\
\text { the well cavity side }\end{array}$ & $d_{2}$ & $d_{4}$ & $d_{2}$ & $d_{4}$ \\
\hline $\begin{array}{l}\theta_{3}<\theta<\theta_{4} \text { and } \varphi>\varphi_{a}{ }^{\prime} \\
\text { the well cavity bottom }\end{array}$ & $d_{1}$ & $d_{3}$ & $d_{1}$ & $d_{3}$ \\
\hline $\begin{array}{l}\theta>\theta_{4} \\
\text { the well cavity side }\end{array}$ & $d_{2}$ & $d_{4}$ & $d_{2}$ & $d_{4}$ \\
\hline
\end{tabular}

Table 1: The path length functions allowed for a photon striking part A of the detector. So, in general the photon path length can follow one of the path lengths d1, d2, d3 and $\mathrm{d} 4$. Table 1 summarizes the faiths that can be followed by the photon.

\begin{tabular}{|c|c|c|c|c|}
\hline $\begin{array}{l}\text { The proton leaves } \\
\text { from }\end{array}$ & $\begin{array}{l}\theta<\theta_{2} \\
\text { bottom }\end{array}$ & $\begin{array}{c}\theta_{2}<\theta<\theta_{6} \\
\varphi<\varphi_{b} \\
\text { bottom }\end{array}$ & $\begin{array}{c}\theta_{2}<\theta<\theta_{6} \\
\varphi>\varphi_{b} \\
\text { side }\end{array}$ & $\begin{array}{r}\theta>\theta_{2} \\
\text { side }\end{array}$ \\
\hline $\begin{array}{l}\theta<\theta_{4} \\
\text { the well cavity bottom }\end{array}$ & $\mathrm{d}_{1}$ & $\mathrm{~d}_{1}$ & $\mathrm{~d}_{3}$ & $\mathrm{~d}_{3}$ \\
\hline $\begin{array}{l}\theta_{4}<\theta<\theta_{5} \text { and } \varphi<\varphi_{a}{ }^{\prime} \\
\text { the well cavity bottom }\end{array}$ & $\mathrm{d}_{1}$ & $\mathrm{~d}_{1}$ & $\mathrm{~d}_{3}$ & $\mathrm{~d}_{3}$ \\
\hline $\begin{array}{l}\theta_{4}<\theta<\theta_{5} \text { and } \varphi>\varphi_{a}{ }^{\prime} \\
\text { the well cavity side }\end{array}$ & $\mathrm{d}_{2}$ & $\mathrm{~d}_{2}$ & $\mathrm{~d}_{4}$ & $\mathrm{~d}_{4}$ \\
\hline $\begin{array}{l}\theta_{5}<\theta \\
\text { the well cavity side }\end{array}$ & $d_{2}$ & $\mathrm{~d}_{2}$ & $\mathrm{~d}_{4}$ & $\mathrm{~d}_{4}$ \\
\hline
\end{tabular}

Table 2: The path length Functions allowed for a photon striking part $B$ of the detector. So, in general the photon path length can follow one of the path lengths d1, d2, d3 and $\mathrm{d} 4$. Table 2 summarizes the faiths that can be followed by the photon.

- For $\theta>\theta_{2}$; the photon must leave from the side.

Also, Figure 8 allows us to derive the equations of $\theta_{1}$ and $\theta_{2}$ :

$$
\begin{aligned}
& \theta_{1}=\tan ^{-1}\left(\frac{R-c}{z}\right) \\
& \theta_{2}=\tan ^{-1} \frac{\sqrt{R^{2}-c^{2}}}{z}
\end{aligned}
$$

Also from Figure 8, it is easy to derive the equation of $\varphi_{\mathrm{a}}$ by applying the cosine law in triangle SOC; we have: $(S O)^{2}+(S C)^{2}+2(S O) .(S C)$. $\cos \left(\varphi_{a}\right)=(O C)^{2}$, which turns after substitution into: $c^{2}+z^{2} \cdot \tan ^{2}(\theta)+$ 2.c.z. $\tan (\theta) \cdot \cos \left(\varphi_{a}\right)=R^{2}$, thus $\varphi_{\mathrm{a}}$ can be done by:

$$
\varphi_{a}=\cos ^{-1}\left(\frac{R^{2}-c^{2}-z^{2} \cdot \tan \theta^{2}}{2 \cdot c \cdot z \cdot \tan \theta}\right)
$$

Now to study how the photon can enter the detector we will make projection on the plane containing the bottom of the well, the projection of the polar angle " $\theta$ " on a horizontal plane is a circle with 


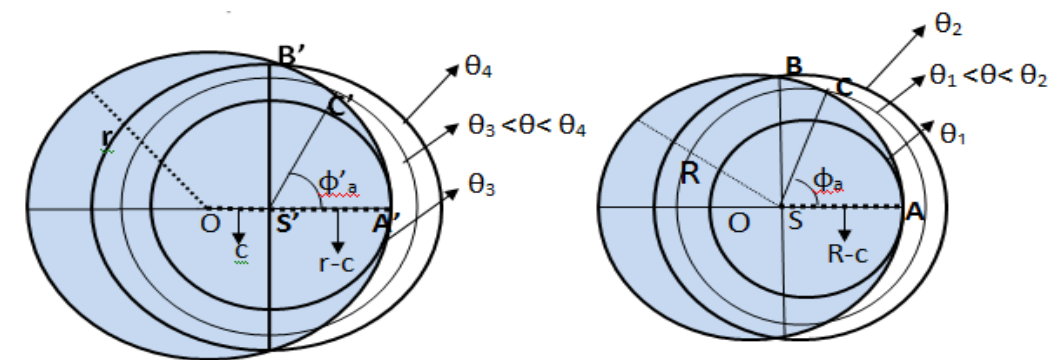

Figure 8: Projection on the horizontal plane containing the well's bottom (left) to show the projection of angles $\theta 3$ and $\theta 4$ and azimuth angle $\varphi a^{\prime}$, and a projection on the detector's bottom (right) to show the projection of angles $\theta 1$ and $\theta 2$ and azimuth angle $\varphi \mathrm{a}$.
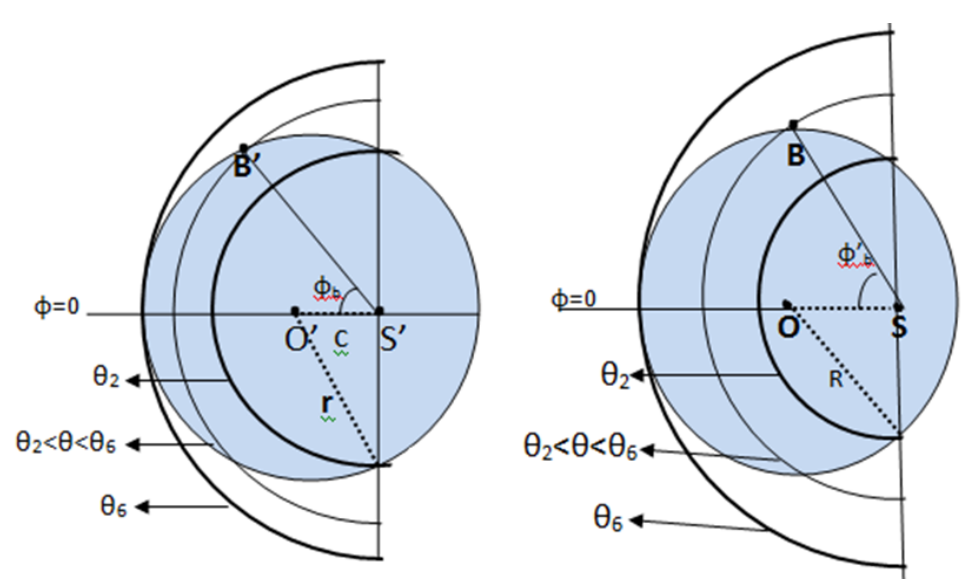

Figure 9: Projection on two horizontal planes (the plane containing the well's bottom to the left and the one containing the detector's bottom to the right) to discuss the path length functions followed by a photon crossing zone $B$ of the detector.

radius [ $\mathrm{f} x \tan (\theta)$ ], where $\mathrm{f}$ designates the height of the source above the plane of projection. As shown in Figure 8; for $\theta<\theta_{3}$ the photon must enter from the bottom of well. But for $\theta_{3}<\theta<\theta_{4}$, the photon can enter from the side if it has an azimuthal angle $\varphi<\varphi_{b}$ For $\theta$ $>\theta_{4}$; the photon must enter from the side. Also from Figure 8 we can derive easily the functions of $\theta_{3}$ and $\theta_{4}$, it is clear: $\sin \left(\theta_{4}\right)=\left(\sqrt{ }\left(r^{2}-c^{2}\right) /\right.$ hypotenuse) while $\cos \left(\theta_{4}\right)=$ (f/ hypotenuse), so

$$
\theta_{4}=\tan ^{-1}\left(\frac{\sqrt{r^{2}-c^{2}}}{f}\right)
$$

and, in a similar manner:

$$
\theta_{3}=\tan ^{-1}\left(\frac{r-c}{f}\right)
$$

In addition we can use Figure 8 to derive the equation of $\varphi_{a}^{\prime}$ by applying the cosine law in triangle $S^{\prime} \mathrm{O}^{\prime} \mathrm{C}^{\prime}$; we have: $\left(S^{\prime} \mathrm{O}^{\prime}\right)^{2}+\left(\mathrm{O}^{\prime} \mathrm{C}^{\prime}\right)^{2}+$ 2. $\left(S^{\prime} O^{\prime}\right) \cdot\left(O^{\prime} C^{\prime}\right) \cdot \cos \left(\varphi_{a}^{\prime}\right)=\left(S^{\prime} C^{\prime}\right)^{2}$, which turns after substitution into: $c^{2}$ $+f^{2} \cdot \tan ^{2}(\theta)+2 \cdot c \cdot f \cdot \tan (\theta) \cdot \cos \left(\varphi_{a}^{\prime}\right)=r^{2}$, thus $\varphi_{a}^{\prime}$ can be done by:

$$
\varphi_{a}^{\prime}=\cos ^{-1}\left(\frac{r^{2}-c^{2}-h^{2} \cdot \tan (\theta)^{2}}{2 . c \cdot f \cdot \tan (\theta)}\right)
$$

Zone B, the further zone with $0<\theta<\pi / 2$ : Just like we studied in zone $\mathrm{A}$, the path length functions followed by the photon in the detector if it strikes zone B will depend on two things only: how it enters and how it leaves the detector material. The photon can enter from either the bottom or the side of the well cavity; we should study the different faiths depending on polar and azimuth angles. Starting by studying the faiths of entrance, we have to make projection on a plane containing the bottom of well cavity, thus according to Figure 9(left), we obtain:
For $\theta<\theta_{4}$; the photon must enter from the bottom, whatever the azimuth angle is $\theta_{4}=\tan ^{-1} \frac{\sqrt{r^{2}-c^{2}}}{h}$ where (as identified before in equation 16)

For $\theta_{4}<\theta<\theta_{5}$ the photon enters the detector from the bottom if it has a small azimuthal angle $\varphi<\varphi_{b}^{\prime}$ or from the side if it has a large azimuthal angle $\varphi>\varphi_{b}^{\prime}$ where:

$$
\theta_{5}=\tan ^{-1}\left(\frac{r+c}{f}\right)
$$

For $\theta>\theta_{5}$; the photon must enter from well's side. While, if we study the faiths the photon will leave the detector according to the polar and azimuthal. For $\theta<\theta_{2}$; the photon must leave from the bottom whatever the azimuth angle is; where $\theta_{2}=\tan ^{-1} \frac{\sqrt{R^{2}-c^{2}}}{z}$ (as identified before
in equation 14);

For $\theta_{2}<\theta<\theta_{6 ;}$ the photon might leave the detector from the bottom if it has a small azimuthal angle $\left(\varphi<\varphi_{\mathrm{b}}\right)$ or from the side if it has a large azimuthal angle $\left(\varphi>\varphi_{\mathrm{b}}\right)$, where

$$
\begin{aligned}
& \theta_{6}=\tan ^{-1}\left(\frac{R+c}{z}\right) \\
& \text { - } \varphi_{\mathrm{b}} \text { is done in equation } 21 \\
& \text { - } \quad \text { For } \theta>\theta_{6} \text {; the photon must leave from the side. }
\end{aligned}
$$

Here, because we are dealing with the bigger part of the detector, so the sign ( \pm ) in the equations $(5-7)$ of $d_{2}, d_{3}$ and $d_{4}$ will turn to (+). By looking at Figure 9, we take the triangle $B S O$ and applying the cosine law in simple mathematics. We have: 
$(O B)^{2}=(O S)^{2}+(S B)^{2}+2 \cdot(S O) \cdot(S B) \cdot \cos \left(\varphi_{b}\right)$, but $(O S)=c,(O B)=r$ and $(S B)=h \tan (\theta)$, so we obtain:

$$
\varphi_{b}=\cos ^{-1}\left(\frac{c^{2}+z^{2} \cdot \tan \theta^{2}-R,^{2}}{2 \cdot c \cdot z \cdot \tan \theta}\right)
$$

Now, it is easy to derive the equation of $\varphi_{b}^{\prime}$, just by applying the same method and taking the triangle $S^{\prime} O^{\prime} B^{\prime}$, we obtain

$$
\varphi_{b}^{\prime} \cos ^{-1}\left(\frac{c^{2}+f^{2} \cdot \tan \theta^{2}-r^{2}}{2 . c . f \cdot \tan \theta}\right)
$$

Zone C: The part of the detector close to the source with $\frac{\pi}{2}<\theta<\pi$ : The particle must enter from the side, while it can leave either from the upper face or the outer side so two path lengths might take place here: $d_{4}$ and $d_{5}$. This depends on the polar and azimuth angles certainly, to study the path followed by the photon according to these angles we can make projection on a horizontal plane but now the horizontal plane will be the one containing the upper face of the detector. Here, unlike zones $\mathrm{A}$ and $\mathrm{B}$, we need projection on one single plane. The projection is shown in Figure 10. By taking the triangle OSY; $O S=c, O Y=r$ and $S Y=(T-z) \cdot \tan (\pi-\theta)$, we can derive the function of $\varphi_{c}$ and by taking the triangle OSX we can derive the equation of $\ddot{o}_{c}^{\prime}$.

If $\theta<\left(\pi-\theta_{7}\right)$, the photon must leave from the side; thus it is going to follow the path length $d_{4}$, where:

$$
\theta_{7}=\tan ^{-1}\left(\frac{\sqrt{\mathrm{R}^{2}-\mathrm{c}^{2}}}{\mathrm{~T}-\mathrm{z}}\right)
$$

If $\left(\pi-\theta_{7}\right)<\theta<\left(\pi-\theta_{8}\right)$, the photon can leave either from upper face if its azimuthal angle is less then certain value we can call it $\varphi_{c}$ or from the side if it has an azimuthal angle $\varphi>\varphi_{c}$, where:

$$
\varphi_{8}=\tan ^{-1}\left(\frac{\mathrm{R}-\mathrm{c}}{\mathrm{T}-\mathrm{z}}\right)
$$

Also, $\varphi_{c}$ can be deduced from Figure 10, and in a way similar to $\varphi_{\mathrm{a}}$ :

$$
\varphi_{c}=\cos ^{-1}\left(\frac{R^{2}-c^{2}-(T-z)^{2} \cdot(\tan (\pi-\theta))^{2}}{2 \cdot c \cdot(T-z) \cdot \tan (\pi-\theta)}\right)
$$

If $\left(\pi-\theta_{8}\right)<\theta<\left(\pi-\theta_{9}\right)$, the photon must leave from the upper face, where:

$$
\theta_{9}=\tan ^{-1}\left(\frac{\sqrt{\mathrm{r}^{2}-\mathrm{c}^{2}}}{\mathrm{~T}-\mathrm{z}}\right)
$$

If $\left(\pi-\theta_{9}\right)<\theta<\left(\pi-\theta_{10}\right)$, the photon can either leave from the upper face if its azimuthal angle is less than certain value we call it $\varphi_{c}$ or escape without hitting the active material of the detector for $\varphi>\varphi_{c}$ where:

$$
\begin{aligned}
& \theta_{10}=\tan ^{-1}\left(\frac{\mathrm{r}-\mathrm{c}}{\mathrm{T}-\mathrm{z}}\right) \\
& \varphi_{c}^{\prime}=\cos ^{-1}\left(\frac{r^{2}-c^{2}-(T-z)^{2} \cdot(\tan (\pi-\theta))^{2}}{2 \cdot c \cdot(T-z) \cdot \tan (\pi-\theta)}\right.
\end{aligned}
$$

If $\theta>\left(\pi-\theta_{10}\right)$, the photon leaves the detector without hitting its active material.

Zone $D$ : The farther part with $\theta>\pi / 2$ : Also for the farther part with polar angle $\theta>\frac{\pi}{2}$; the photon must enter from the side while it can leave either from ${ }^{2}$ side or upper face. We should study whether the photon will leave from the upper face or from the side by making a projection on the plane containing the upper face of detector just like we did in the previous part. The projection is shown in Figure 11.

- If $\theta<\left(\pi-\theta_{11}\right)$, the photon must leave from the side and the only path way followed is $d_{4}$, where:

$\theta_{11}=\tan ^{-1}\left(\frac{\mathrm{R}+\mathrm{c}}{\mathrm{T}-\mathrm{z}}\right)$

- If $\left(\pi-\theta_{11}\right)<\theta<\left(\pi-\theta_{7}\right)$, the photon can leave either from side or upper face depending on the azimuthal angle. It leaves from upper face for azimuthal angle $\varphi<\varphi_{\mathrm{d}}$ and it leaves from side if $\varphi>\varphi_{\mathrm{d}}$.

- If $\left(\pi-\theta_{7}\right)<\theta<\left(\pi-\theta_{12}\right)$, the photon must leave from the upper face whatever the azimuthal angle was, where:

$\theta_{12}=\tan ^{-1}\left(\frac{\mathrm{r}+\mathrm{c}}{\mathrm{T}-\mathrm{z}}\right)$

If $\left(\pi-\theta_{12}\right)<\theta<\left(\pi-\theta_{9}\right)$, the photon can escape without striking the material of detector for $\varphi<\varphi_{d}^{\prime}$ or strike it entering from the side and leaving from the face if $\varphi=\varphi_{d}^{\prime}$.

- For $\theta>\left(\pi-\theta_{9}\right)$, the photon cannot strike the detector.

Also, Figure 11 helps to derive the equations of the new azimuthal angles: $\varphi_{d}$ and $\phi_{\mathrm{d}}$ in a manner very similar to $\phi_{\mathrm{c}}$ and $\varphi_{c}$.

$$
\begin{aligned}
& \cos \left(\varphi_{d}^{\prime}\right)=\frac{(T-z)^{2} \cdot \tan (\theta)^{2}+c^{2}-r^{2}}{(T-z) \cdot \tan \theta \cdot c} \\
& \left(\varphi_{d}\right)=\frac{(T-z)^{2} \cdot \tan (\theta)^{2}+c^{2}-R^{2}}{(T-z) \cdot \tan \theta \cdot c}
\end{aligned}
$$

Thus the efficiency of the detector for a non-axial point source will be done by:

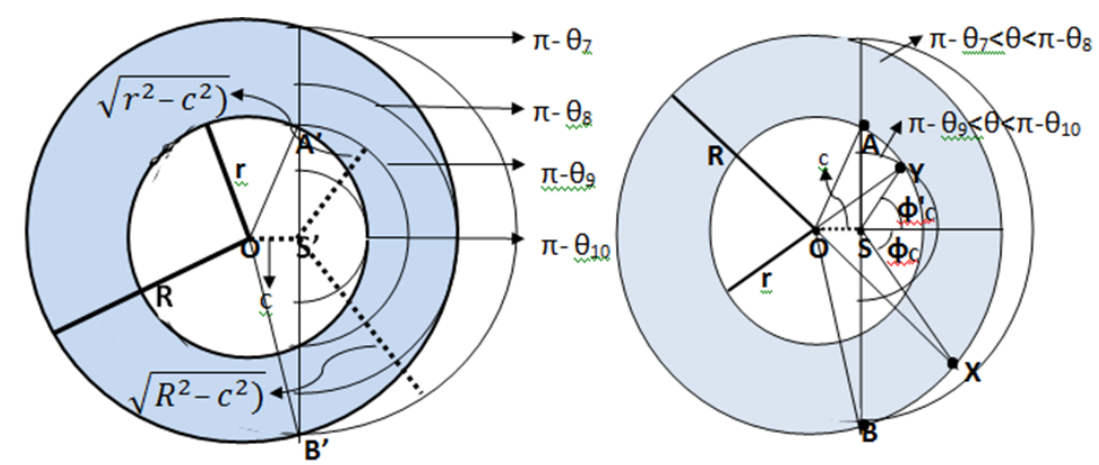

Figure 10: Projection to a horizontal plane containing the surface of detector the right figure can be used to determine the equations of $\varphi_{\mathrm{c}}$ and $\varphi_{\mathrm{c}}$. 

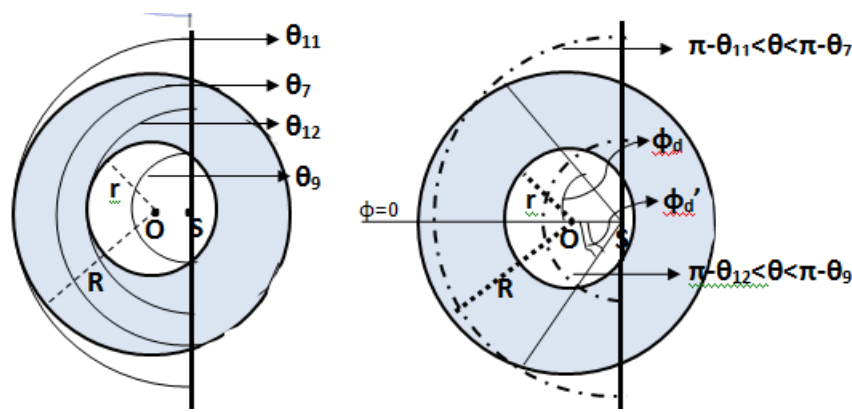

Figure 11: Projection on the upper plane of the detector.

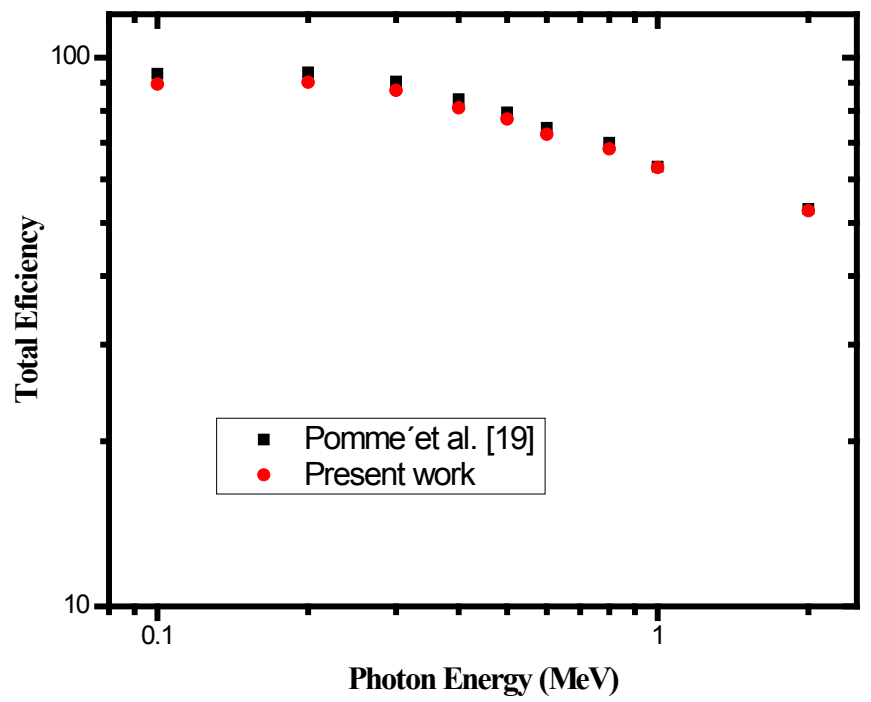

Figure 12: Shows the present work and Monte Carlo [19] values for an axial point source positioned at height $z=8.55 \mathrm{~cm}$, from the well bottom of detector IRMM, without source-walls.

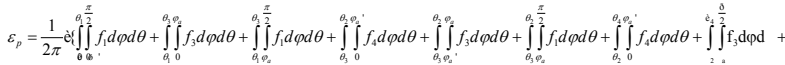

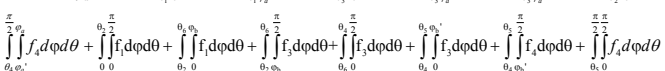

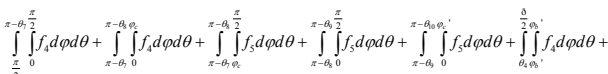

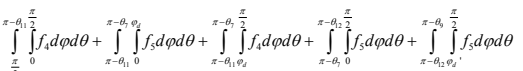

Where, $f$ is as identified before in equation 2 . The previous equation can be used for $\theta_{3}<\theta_{2}$ and $\theta_{6}<\theta_{4}$.

\section{Absolute Efficiency for Disk and Cylindrical Sources}

The efficiency of a circular disk source of radius $S$ can be done by:

$$
\varepsilon_{d}=\frac{2}{S^{2}} \int_{0}^{S} \varepsilon_{P} \cdot c d c
$$

Where $\varepsilon_{p}$ is the efficiency of a non-axial point source, as identified before in equation (33).In the case of cylindrical sources, we can calculate the efficiency by making an integration of $\varepsilon_{p}$ over $d c$ and $d_{h}$ and taking into consideration the efficiencies of many point sources on the same shell are equals. So for a cylinder of radius $S$ and height $H$ we have:

$$
\varepsilon_{c y l}=\frac{2}{S^{2} \times H} \int_{0}^{H} \int_{0}^{S} \varepsilon_{P} \cdot c d c d h
$$

\section{Results and Comparisons}

In this study we used two well type detectors [15], (1) IRMM is used in Figures 12-17 and (2) CIEMAT is used in Figure 18. The dimensions of the two detectors are given in Table 3 . The following figures illustrate the comparison between the theoretical calculated values (present work) and the published Monte Carlo values for different sources. The percentage deviation is given by:

$$
\Delta=\frac{\varepsilon_{\text {expt. }}-\varepsilon_{\text {theo. }}}{\varepsilon_{\text {expt. }}} \times 100 \%
$$

where, $\boldsymbol{\varepsilon}_{\text {theo }}$ and $\boldsymbol{\varepsilon}_{\text {expt }}$ are the theoretical (present work) and the Monte Carlo efficiencies, respectively [17-21]

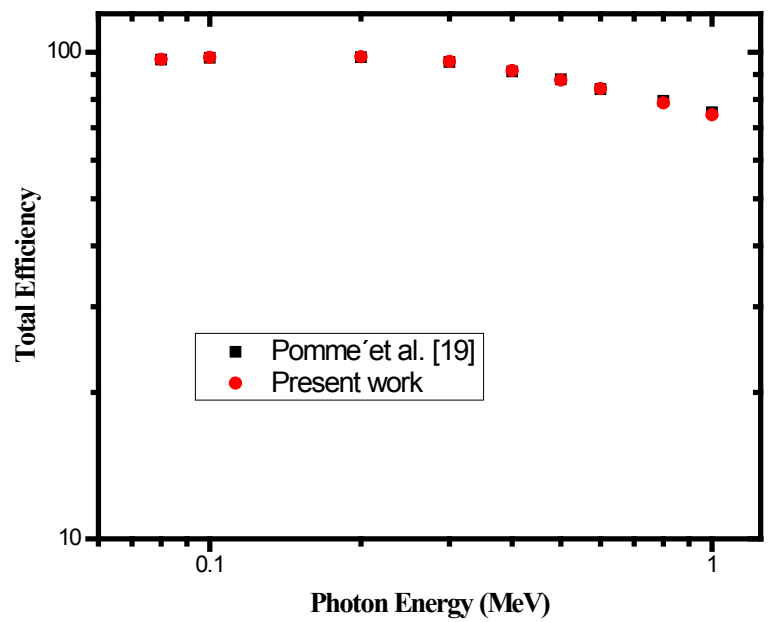

Figure 13: Shows the present work and Monte Carlo [19] values for a nonaxial point source positioned at height $z=5.66 \mathrm{~cm}$, from the well bottom of detector IRMM, without source-walls.

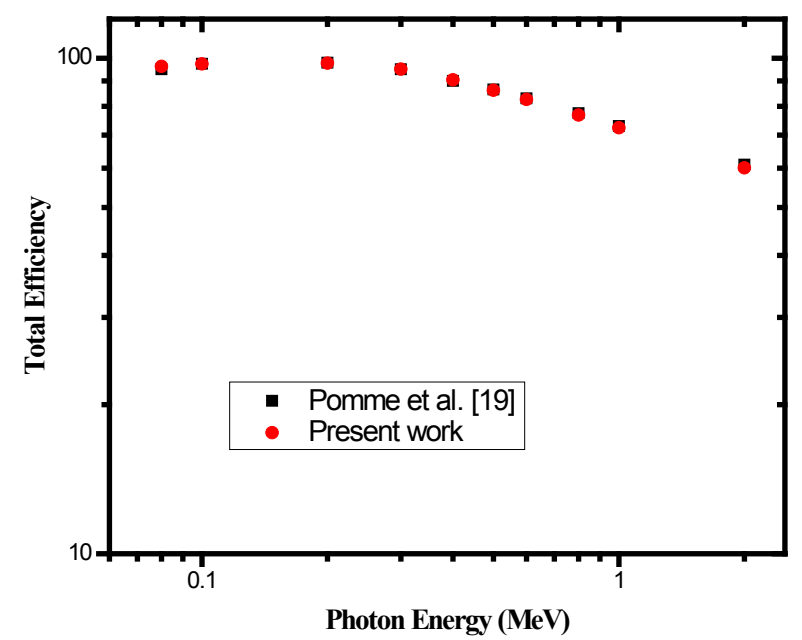

Figure 14: Shows the present work and Monte Carlo [19] values an eccentric cylindrical source, with $2 \mathrm{~cm}$ radius and $4 \mathrm{~cm}$ height, located at $z=6.05 \mathrm{~cm}$, from the well bottom of detector IRMM, within a thick Acrylic container with walls (bottom and surface) of $0.1 \mathrm{~cm}$ thickness. 


\section{Conclusions}

The present approach offers straightforward mathematical equations to calibrate well-type $\mathrm{NaI}(\mathrm{Tl})$ scintillation detector over a wide energy range. This approach is based on simple mathematical integrals and computer programs to calculate the detection efficiency. It does not need neither optimization of the detector parameters or previous knowledge of a standard source values. The comparison between calculated and reference values shows that the difference is very small (never escaped $8 \%$ and reduced to less than $2 \%$ in most cases).

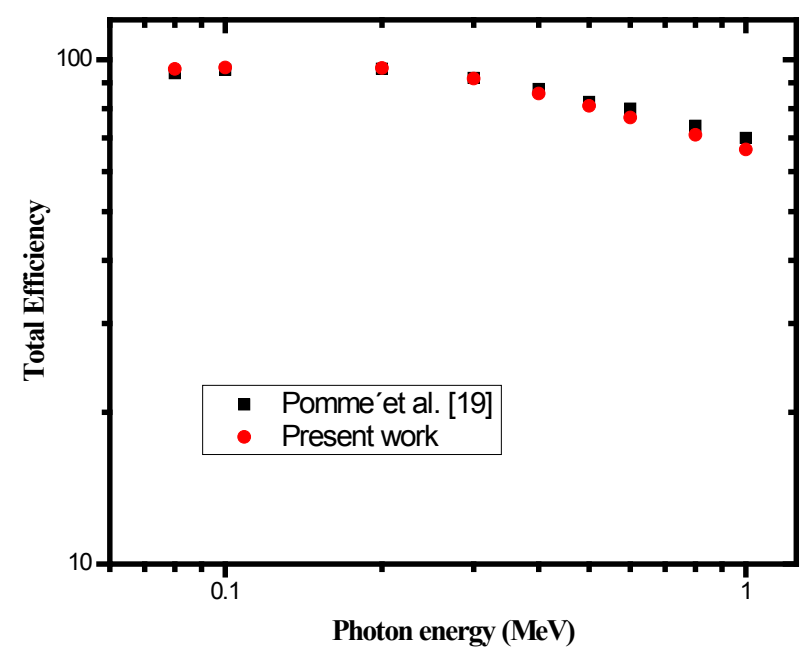

Figure 15: Shows the present work and Monte Carlo [19] values for a coaxial cylindrical source, with $2 \mathrm{~cm}$ radius and $4 \mathrm{~cm}$ height, and its bottom is located at a height $z=6.05 \mathrm{~cm}$, from the well bottom of detector IRMM, within a thick Acrylic container with walls (bottom and surface) of $0.1 \mathrm{~cm}$ thickness.

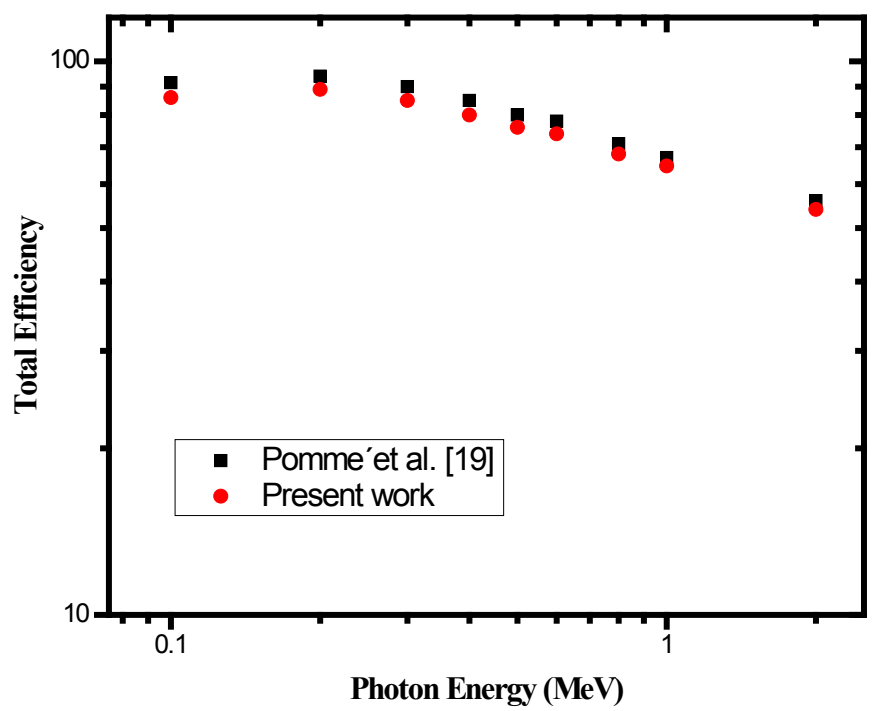

Figure 16: Shows the present work and Monte Carlo [19] values for an axial point source located at a height $z=5.55 \mathrm{~cm}$, from the well bottom of detector IRMM, within a thick Acrylic container with walls (bottom and surface) of 2.5 $\mathrm{cm}$ thickness.

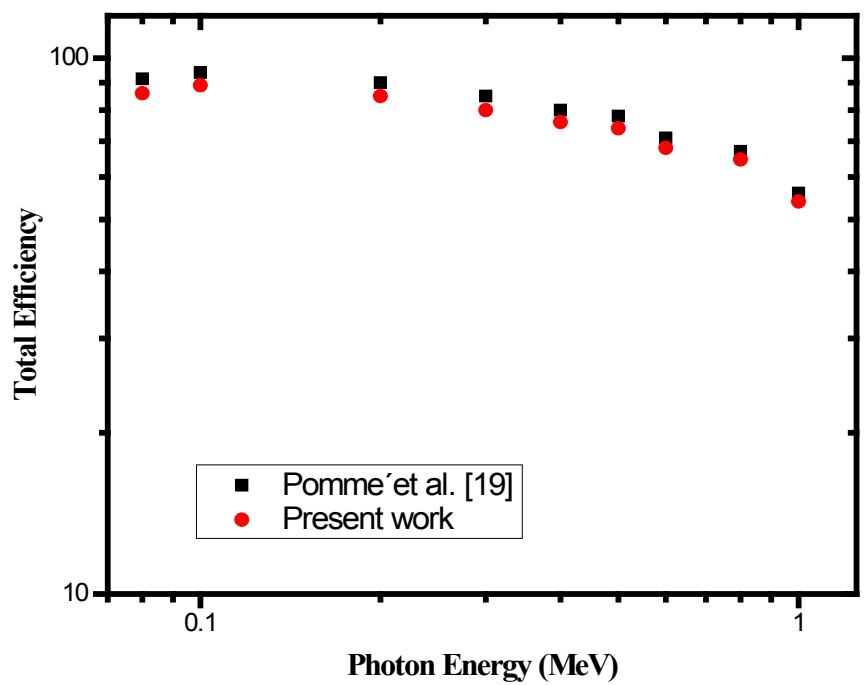

Figure 17: Shows the present work and Monte Carlo [19] values for an axial point source located at a height $z=7.85 \mathrm{~cm}$, from the well bottom of detector IRMM, within an Aluminum container with walls of $1 \mathrm{~cm}$ thickness and its bottom and surface of thickness $0.2 \mathrm{~cm}$.

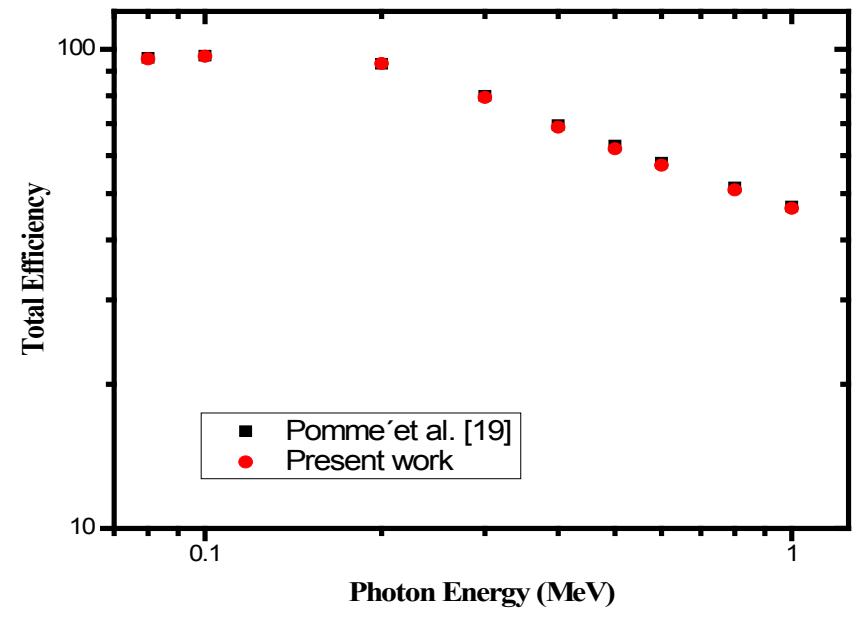

Figure 18: Shows the present work and Monte Carlo [19] values for a coaxial disk source, with radius $=0.15 \mathrm{~cm}$, located at a height $\mathrm{z}=2.61 \mathrm{~cm}$, from the well bottom of detector CIEMAT, within a very thin Acrylic container.

\begin{tabular}{|c|c|c|}
\hline & IRMM & CEIMAT \\
\hline The detector radius (R) & 7.62 & 3.81 \\
\hline The well radius ( $r$ ) & 2.62 & 1.45 \\
\hline The entire height $(T)$ & 15.23 & 7.62 \\
\hline Bottom to well distance $(\mathrm{H})$ & 5.43 & 2.54 \\
\hline $\begin{array}{l}\text { Well's cover thickness Made of Aluminum and } \\
\text { Aluminum Oxide }\end{array}$ & 0.12 & 0.06 \\
\hline Well's bottom & 0.12 & 0.06 \\
\hline
\end{tabular}

Table 3: Shows the dimensions (are in $\mathrm{cm}$ ) of the two well-type detectors. 
Citation: Abbas MI, Ibrahim OA, Ibrahim T, Sakr M (2016) New Analytical Formulae to Calibrate Well-type Scintillation Detectors Efficiency. J Phys Math 7: 164. doi:10.4172/2090-0902.1000164

\section{References}

1. Selim YS, Abbas MI (1996) Direct Calculation of the total efficiency of cylindrical scintillation detectors for extended circular sources. Radiation physics and chemistry 48: 23-27.

2. Selim YS, Abbas MI, Fawzy MA (1998) Analytical calculation of the efficiencies of gamma scintillators efficiencies. I: total efficiency for coaxial disk sources. Radiat Phys Chem 53: 589-592.

3. Selim YS, Abbas MI (2000) Analytical calculations of gamma scintillators efficiencies. II: total efficiency for wide co-axial disk sources. Radiat Phys Chem 53: $15-19$

4. Abbas MI (2001) HPGe detector photopeak efficiency calculation including self-absorption and coincidence corrections for Marinilli beaker sources using compact analytical expressions. Appl Radiat Isoto 54: 761-768.

5. Abbas MI (2001) A direct mathematical method to calculate the efficiencies of a parallelepiped detector for an arbitrarily positioned point source. Radiat Phys Chem 60: 03-09.

6. Abbas MI (2001)Analytical formulae for well-type $\mathrm{Nal}(\mathrm{TI})$ and HPGe detectors efficiency computation. Appl Radiat Isot 55: 245-252.

7. Abbas MI Selim YS, Bassiouni M (2001) HPGe Detector Photopeak Efficiency Calculation including Self-Absorption and Coincidence Corrections for Cylindrical Sources using Compact Analytical Expressions. Radiat Phys Chem 61: 429-431.

8. Abbas MI Selim, YS (2002) Calculation of relative full-energy peak efficiencies of well-type detectors. Nucl Instrum Meth A 480: 651-657.

9. Abbas MI (2006) Analytical calculations of the solid angles subtended by a well-type detector at a point and extended circular source. Appl Radiat Isot 64 1048-1056.

10. Abbas MI, Nafee S, Selim YS (2006) Calibration of cylindrical detectors using a simplified theoretical approach. Appl Radiat Isot 64: 1057-1064.

11. Abbas MI (2011) A new analytical method to calibrate cylindrical phoswich and $\mathrm{LaBr}_{3}(\mathrm{Ce})$ scintillation detectors. Nucl Instrum Meth A 621: 413-418.
12. Abbas MI (2010) Analytical formulae for borehole scintillation detectors efficiency calibration. Nucl Instrum Meth A 622: 171-175.

13. Grosswendt B, Waibel E (1976) Monte Carlo calculation of the intrinsic gamma ray efficiencies of cylindrical $\mathrm{Nal}(\mathrm{TI})$ detectors. Nucl Instrum Methods 133: 2528.

14. Hernandez F, El-Daoushy F (2003) Accounting for incomplete charge collection in Monte Carlo simulations of the efficiency of well-type Ge-detectors. Nuc Instrum Meth A 498: 340-351.

15. Pomme' S, Sibbens G, Vidmar T, Camps J, Peyres V (2009) Detection efficiency calculation for photons, electrons and positrons in a well detector. Part II: Analytical model versus simulations. Nuclear Instruments and Methods in Physics Research A 606: 501-507.

16. Ahmed AH (2003) Accurate Direct Mathmatical Determination of The Efficiencies Of Gamma Detectors Arising From Radioactive Sources O Different Shapes. Alexandria University.

17. Blaauw M (1993) To use of Sources Emitting Coincident y-rays for Determination of Absolute Efficiency Curves of Highly Efficient Ge Detectors. Nucl Instrum Meth A 332: 493-500.

18. Sima O (2000) Accurate calculation of total efficiency of Ge well-type detectors suitable for efficiency calibration using common standard sources. Nucl Instrum Meth A 450: 98-108.

19. Badawi MS, El-Khatib AM, Krar ME (2013) New Numerical Simulation Approach to Calibrate the Nal(TI) Detectors Array Using Non-Axial Extended Spherical Sources. Journal of Instrumentation 8: 11-20.

20. Badawi MS, Elzaher MA, Thabet AA, El-khatib, AM (2013) An empirical formula to calculate the full energy peak efficiency of scintillation detectors. Appl Radiat Isot 74: 46-49.

21. L'Annunziata MF (2011) Handbook of Radioactivity Analysis. (2ndedn) Academic press. 